\title{
The sampling theorem and coherent state systems in quantum mechanics
}

\author{
Arvind $^{1}$, S Chaturvedi ${ }^{2}$, N Mukunda ${ }^{3}$ and R Simon ${ }^{4}$ \\ ${ }^{1}$ Department of Physics, Indian Institute of Technology Madras, Chennai 600036, India \\ ${ }^{2}$ School of Physics, University of Hyderabad, Hyderabad 500046, India \\ ${ }^{3}$ Centre for High Energy Physics, Indian Institute of Science, Bangalore 560012, India \\ ${ }^{4}$ The Institute of Mathematical Sciences, C.I.T. Campus, Chennai 600113, India \\ E-mail: arvind@physics.iitm.ac.in, scsp@uohyd.ernet.in,nmukunda@cts.iisc.ernet.in \\ and simon@imsc.res.in
}

Received 21 February 2006

Accepted for publication 6 April 2006

Published 18 July 2006

Online at stacks.iop.org/PhysScr/74/168

\begin{abstract}
The well-known Poisson Summation Formula is analysed from the perspective of the coherent state systems associated with the Heisenberg-Weyl group. In particular, it is shown that the Poisson Summation Formula may be viewed abstractly as a relation between two sets of bases (Zak bases) arising as simultaneous eigenvectors of two commuting unitary operators in which geometric phase plays a key role. The Zak bases are shown to be interpretable as generalized coherent state systems of the Heisenberg-Weyl group and this, in turn, prompts analysis of the sampling theorem (an important and useful consequence of the Poisson Summation Formula) and its extension from a coherent state point of view leading to interesting results on the properties of von Neumann and finer lattices based on standard and generalized coherent state systems.
\end{abstract}

PACS number: 03.65.-w

\section{Introduction}

It is well known that the Sampling Theorem (ST) for band-limited signals [1], of fundamental importance in communication theory, arises from the properties of the Fourier transform operation on the real line, and the related Poisson Summation Formula. It is also well known that the Heisenberg-Weyl (H-W) group [2], which is basic for nonrelativistic quantum kinematics, is intimately related to the same Fourier transformation. This is clear from the description of particle momentum in wave mechanics and in the position-momentum uncertainty principle. As will become evident, it is possible to derive the Poisson Summation Formula in a particularly elegant manner from the representation theory of the H-W group.

Many applications of this group use the remarkable properties of the so-called 'coherent states' originally discovered by Schrödinger [3], and extensively used in quantum optics in particular [4]. The theory of these and other systems of coherent states, called 'generalized coherent states', has been put on a comprehensive footing, and the extension to such systems associated with general Lie groups has been carried out [5]. In the process, it has been realized that even for a given Lie group, such as the H-W group, one can construct many different systems of generalized coherent states, sharing some features dictated by the structure of the group, but differing from one another in certain details.

These remarks suggest that the $\mathrm{H}-\mathrm{W}$ group functions as a unifying element or as a common connecting thread linking various ideas and concepts, each of which figuratively flows out of the group and its representations in a different direction-Poisson Summation Formula, ST, specific families of generalized coherent states and, as one finds, even certain instances of the recently much studied geometric phase. ${ }^{5}$ There is yet another sense in which the usual ST and the standard coherent states share some common features. There are certain discrete subsets of the coherent states, namely the so-called von Neumann lattice of these states and finer lattices, which enjoy the property of 'totality' or (over) completeness in the relevant Hilbert space: any vector in this space is in principle fully determined once one knows its inner products with all the vectors in the lattice [7]. Evidently,

\footnotetext{
5 A comprehensive account of the geometric phase and original reprints may be found in [6].
} 
this too is in a sense a ST. These lattices of states and some generalizations have been studied extensively some time ago, developing in the process simpler proofs of totality, analysis of conditions leading to orthonormality etc [8]. It would seem to be of considerable interest to express the usual ST in such a way that a comparison with the properties of lattices of coherent states, standard or generalized, could be easily carried out.

In this work we attempt to forge a certain sense of unity among these various concepts from the perspective of coherent state systems of the $\mathrm{H}-\mathrm{W}$ group and seek extensions and generalizations of known results to the possible extent. A brief outline of this work is as follows. In section 2 we recapitulate features of the $\mathrm{H}-\mathrm{W}$ group to the extent required in this work and show how the Poisson Summation Formula arises as a consequence of the relation between two bases consisting of two commuting unitary operators and highlight the role the geometric phase plays in this context. In section 3, we establish connection between the two bases and the Zak representation [9] and further show that the two can be identified with certain generalized coherent states of the H-W group. We also discuss some of their special features needed later and in section 4 give the Wigner distribution of the underlying fiducial vector. Section 5 is devoted to two forms of the standard ST for band-limited state vectors. In section 6 we translate the contents of the standard ST into the properties of standard coherent state lattices and extend the results to a general state vector and compare them with known results on von Neumann and finer standard coherent state lattices. Similar questions in the context of generalized coherent state systems are explored in section 7. Section 8 contains concluding remarks and further outlook.

\section{The H-W group and the Poisson Summation Formula}

The H-W group and its associated operator structures are based on the fundamental Heisenberg canonical commutation relation

$$
[\hat{q}, \hat{p}]=\mathrm{i},
$$

for hermitian operators $\hat{q}, \hat{p}$ representing position and momentum respectively for a one-dimensional Cartesian quantum mechanical system. (For simplicity we set Planck's constant $\hbar=1$.) Thus, this group is a three parameter Lie group whose elements and composition law may be written as follows:

$$
\begin{gathered}
D\left(\alpha_{1}, \alpha_{2}, \alpha_{3}\right)=\exp \left\{-\mathrm{i} \alpha_{1} \hat{p}+\mathrm{i} \alpha_{2} \hat{q}-\mathrm{i} \alpha_{3}\right\}, \\
-\infty<\alpha_{1}, \alpha_{2}<\infty, \quad 0 \leqslant \alpha_{3}<2 \pi, \\
\quad D\left(\alpha_{1}^{\prime}, \alpha_{2}^{\prime}, \alpha_{3}^{\prime}\right) D\left(\alpha_{1}, \alpha_{2}, \alpha_{3}\right) \\
=D\left(\alpha_{1}^{\prime}+\alpha_{1}, \alpha_{2}^{\prime}+\alpha_{2}, \alpha_{3}^{\prime}+\alpha_{3}+\frac{1}{2}\left(\alpha_{1}^{\prime} \alpha_{2}-\alpha_{2}^{\prime} \alpha_{1}\right)\right) .
\end{gathered}
$$

(In the element on the right, the final phase is understood to be taken modulo $2 \pi$.) According to the Stone-von
Neumann theorem [10] there is essentially only one nontrivial unitary irreducible representation of this group, i.e., only one irreducible hermitian representation of the commutation relation (2.1), apart from unitary equivalence. We shall write $\mathcal{H}$ for the Hilbert space of this representation.

The displacement operators correspond to setting $\alpha_{3}=0$ and to taking $\left(\alpha_{1}, \alpha_{2}\right)$ to be a point $(q, p)$ in the classical phase space or plane:

$D(q, p)=\exp \{\mathrm{i} p \hat{q}-\mathrm{i} q \hat{p}\}, \quad-\infty<q, p<\infty$.

Their basic properties are read off from equation (2.2):

$$
D(q, p)^{-1}=D(q, p)^{\dagger}=D(-q,-p),
$$

$$
D\left(q^{\prime}, p^{\prime}\right) D(q, p)=\exp \left\{\frac{\mathrm{i}}{2}\left(p^{\prime} q-q^{\prime} p\right)\right\} D\left(q^{\prime}+q, p^{\prime}+p\right)
$$

$$
D(q, p)^{-1}(\hat{q} \text { or } \hat{p}) D(q, p)=\hat{q}+q \text { or } \hat{p}+p .
$$

When $q$ or $p$ vanishes it is convenient to define

$$
\begin{aligned}
& U(p)=D(0, p)=\mathrm{e}^{\mathrm{i} p \hat{q}}, \\
& V(q)=D(q, 0)=\mathrm{e}^{-\mathrm{i} q \hat{p}} .
\end{aligned}
$$

For these we have the useful relations

$$
\begin{aligned}
D(q, p) & =\mathrm{e}^{\mathrm{i} q p / 2} V(q) U(p), \\
& =\mathrm{e}^{-\mathrm{i} q p / 2} U(p) V(q), \\
U(p) V(q) & =\mathrm{e}^{\mathrm{i} q p} V(q) U(p) .
\end{aligned}
$$

This last relation for the unitary operators $U(p), V(q)$ is just the finite Weyl form of the commutation relation (2.1); the phase factor present here is the geometric phase associated with the $\mathrm{H}-\mathrm{W}$ group.

Let us denote the usual delta function normalized ideal eigenvectors of $\hat{q}$ and $\hat{p}$, which form continuous bases for $\mathcal{H}$, by angular and rounded ket vectors respectively:

$$
\begin{gathered}
\hat{q}|q\rangle=q|q\rangle, \quad \hat{p} \mid p)=p \mid p), \quad q, p \in \mathcal{R}, \\
\left\langle q^{\prime} \mid q\right\rangle=\delta\left(q^{\prime}-q\right), \quad\left(p^{\prime} \mid p\right)=\delta\left(p^{\prime}-p\right), \\
\langle q| p)=\frac{1}{\sqrt{2 \pi}} \mathrm{e}^{\mathrm{i} q p}
\end{gathered}
$$

On these the actions of the exponentiated unitary operators are:

$$
\begin{gathered}
V(q)\left|q^{\prime}\right\rangle=\left|q^{\prime}+q\right\rangle, \\
D(q, p)\left|q^{\prime}\right\rangle=\mathrm{e}^{\mathrm{i} p\left(q^{\prime}+q / 2\right)}\left|q^{\prime}+q\right\rangle, \\
\left.\left.U(p) \mid p^{\prime}\right)=\mid p^{\prime}+p\right), \\
\left.\left.D(q, p) \mid p^{\prime}\right)=\mathrm{e}^{-\mathrm{i} q\left(p^{\prime}+p / 2\right)} \mid p^{\prime}+p\right) .
\end{gathered}
$$

The operators $U(p)$ and $V(q)$ do not commute in general. Now choose some real positive $q_{0}$ and write

$$
V_{0}=V\left(q_{0}\right)=\mathrm{e}^{-\mathrm{i} q_{0} \hat{p}}
$$


We ask for the smallest nontrivial value of $p$ in $U(p)$, assumed positive, such that $U(p)$ commutes with $V_{0}$ : this happens for $p=2 \pi / q_{0}$, so we define

$$
U_{0}=U\left(2 \pi / q_{0}\right)=\mathrm{e}^{2 \pi \mathrm{i} \hat{q} / q_{0}},
$$

and then have

$$
U_{0} V_{0}=V_{0} U_{0}
$$

It is important to observe that both unitary operators $U_{0}$ and $V_{0}$ are determined by the single parameter $q_{0}$.

We look for the simultaneous (ideal) eigenvectors of $U_{0}$ and $V_{0}$. Their eigenvalues are phases that can be parametrized as follows:

$$
\begin{gathered}
U_{0} \rightarrow \mathrm{e}^{2 \pi \mathrm{i} q / q_{0}}, \quad q \in\left[-\frac{1}{2} q_{0}, \frac{1}{2} q_{0}\right], \\
V_{0} \rightarrow \mathrm{e}^{-\mathrm{i} q_{0} p}, \quad p \in\left[-\pi / q_{0}, \pi / q_{0}\right] .
\end{gathered}
$$

Given a pair $(q, p)$ within these limits, i.e., a point in the rectangle $R\left(q_{0}\right)$ in the phase plane with sides $q_{0}, 2 \pi / q_{0}$ centred at the origin, we can build a simultaneous (ideal) eigenvector of $U_{0}$ and $V_{0}$ either in the $|q\rangle$ basis or in the $\mid p$ ) basis. For this we need to use the actions (2.8) of $U$ and $V$ on these bases. In this way we find after elementary algebra:

$$
\begin{gathered}
|q, p\rangle=\frac{q_{0}^{1 / 2}}{\sqrt{2 \pi}} \sum_{n \in \mathcal{Z}} \mathrm{e}^{\mathrm{i} n q_{0} p}\left|q+n q_{0}\right\rangle, \\
\left(U_{0} \text { or } V_{0}\right)|q, p\rangle=\left(\mathrm{e}^{2 \pi \mathrm{i} q / q_{0}} \text { or } \mathrm{e}^{-\mathrm{i} q_{0} p}\right)|q, p\rangle, \\
\left\langle q^{\prime}, p^{\prime} \mid q, p\right\rangle=\delta\left(q^{\prime}-q\right) \delta\left(p^{\prime}-p\right) .
\end{gathered}
$$

This construction started from the eigenvectors $|q\rangle$ of $\hat{q}$. Alternatively, we can build the simultaneous eigenvectors starting from the basis $\mid p$ ). Then we find:

$$
\begin{gathered}
\left.\mid q, p)=q_{0}^{-1 / 2} \sum_{n \in \mathcal{Z}} \mathrm{e}^{-2 \pi \mathrm{i} n q / q_{0}} \mid p+2 \pi n / q_{0}\right), \\
\left.\left.\left(U_{0} \text { or } V_{0}\right) \mid q, p\right)=\left(e^{2 \pi \mathrm{i} q / q_{0}} \text { or } \mathrm{e}^{-\mathrm{i} q_{0} p}\right) \mid q, p\right), \\
\left(q^{\prime}, p^{\prime} \mid q, p\right)=\delta\left(q^{\prime}-q\right) \delta\left(p^{\prime}-p\right) .
\end{gathered}
$$

We expect that these two solutions must be phase related. We easily find:

$$
\left.\left\langle q^{\prime}, p^{\prime}\right| q, p\right)=\mathrm{e}^{\mathrm{i} q p} \delta\left(q^{\prime}-q\right) \delta\left(p^{\prime}-p\right),
$$

which implies

$$
\mid q, p)=\mathrm{e}^{\mathrm{i} q p}|q, p\rangle .
$$

We will recognize in the next section that this phase is the same H-W geometric phase already present in equation (2.6).

The relation (2.16) in conjugate form is

$$
\begin{gathered}
\frac{q_{0}^{1 / 2}}{\sqrt{2 \pi}} \sum_{n \in \mathcal{Z}} \mathrm{e}^{-\mathrm{i} n q_{0} p}\left\langle q+n q_{0}\right| \\
=q_{0}^{-1 / 2} \mathrm{e}^{\mathrm{i} q p} \sum_{n \in \mathcal{Z}} \mathrm{e}^{2 \pi \mathrm{i} n q / q_{0}}\left(p+2 \pi n / q_{0} \mid .\right.
\end{gathered}
$$

Let $|\psi\rangle \in \mathcal{H}$ be a general normalizable vector with position and momentum space wavefunctions $\psi(q), \varphi(p)$ respectively:

$$
\begin{aligned}
& \psi(q)=\langle q \mid \psi\rangle=\frac{1}{\sqrt{2 \pi}} \int_{-\infty}^{\infty} \mathrm{d} p \varphi(p) \mathrm{e}^{\mathrm{i} p q}, \\
& \varphi(p)=\left(p|\psi\rangle=\frac{1}{\sqrt{2 \pi}} \int_{-\infty}^{\infty} \mathrm{d} q \psi(q) \mathrm{e}^{-\mathrm{i} q p} .\right.
\end{aligned}
$$

Then taking the products of the two sides of equation (2.17) with $|\psi\rangle$ and reinstating the parameter ranges we get:

$$
\begin{gathered}
q_{0} \sum_{n \in \mathcal{Z}} \mathrm{e}^{-\mathrm{i} n q_{0} p} \psi\left(q+n q_{0}\right) \\
=\sqrt{2 \pi} \mathrm{e}^{\mathrm{i} q p} \sum_{n \in \mathcal{Z}} \mathrm{e}^{2 \pi \mathrm{i} n q / q_{0}} \varphi\left(p+2 \pi n / q_{0}\right), \\
q_{0}>0, \quad(q, p) \in R\left(q_{0}\right) .
\end{gathered}
$$

This is the Poisson Summation Formula for any Fourier transform pair $\psi(q), \varphi(p) .{ }^{6}$ It is usually derived quite directly from the structure of the Fourier Series representation for a function of an angle variable, by extending it to a periodic function on the full real line. We see here that it arises very naturally in a quantum mechanical context by constructing simultaneous eigenvectors of the commuting unitary operators $U_{0}, V_{0}$ in two ways and relating the results. This brings out the connection to the $\mathrm{H}-\mathrm{W}$ group. We also see that extending $(q, p)$ in equation (2.19) outside $R\left(q_{0}\right)$ does not give any additional information.

\section{Connection to Zak representation as a generalized coherent state system}

The simultaneous (ideal) eigenvectors of the commuting unitary operators $U_{0}$ and $V_{0}$ developed in two ways in the previous section lead to new representations of vectors $|\psi\rangle \in$ $\mathcal{H}$, distinct from the representations based on position and momentum wavefunctions $\psi(q)$ and $\varphi(p)$. These are the Zak representations of quantum mechanics [16], known and studied for a long time and exploited in particular to examine the von Neumann lattice of standard coherent states and its generalizations [8]. The states $|q, p\rangle, \mid q, p)$ of equations (2.13) and (2.14) are in fact the Zak basis states for $\mathcal{H}$. We explore briefly in this section the possibility of interpreting them as a system of (ideal) generalized coherent states associated with the H-W group. First we begin with the Zak representation in quantum mechanics.

Given $|\psi\rangle \in \mathcal{H}$ with conventional wavefunctions $\psi(q), \varphi(p)$ where $q, p \in \mathcal{R}$, we define the Zak wavefunction $\chi(q, p)$ of $|\psi\rangle$ by

$$
\begin{aligned}
\chi(q, p) & =\langle q, p \mid \psi\rangle, \\
& =\frac{q_{0}^{1 / 2}}{\sqrt{2 \pi}} \sum_{n \in \mathcal{Z}} \mathrm{e}^{-\mathrm{i} n q_{0} p} \psi\left(q+n q_{0}\right) .
\end{aligned}
$$

6 Strictly speaking, this relation does not hold for arbitrary Lebesgue square integrable wavefunctions $\psi(q), \varphi(p)$ since these are defined only upto sets of measure zero. One must limit oneself to, say, the subset of continuous wavefunctions. These qualifications will be understood in the following. 
(Here of course $q_{0}$ is a positive parameter chosen freely and then held fixed.) This definition is based on equation (2.13). Equally well we can use equation (2.14) and define

$$
\begin{aligned}
\tilde{\chi}(q, p) & =(q, p|\psi\rangle, \\
& =\mathrm{e}^{-\mathrm{i} q p} \chi(q, p), \\
& =q_{0}^{-1 / 2} \sum_{n \in \mathcal{Z}} \mathrm{e}^{2 \pi \mathrm{i} n q / q_{0}} \varphi\left(p+2 \pi n / q_{0}\right) .
\end{aligned}
$$

In both equations (3.1) and (3.2), it is understood that $(q, p) \in$ $R\left(q_{0}\right)$. These equations define the so-called Zak transform, and exhibit the Hilbert space $\mathcal{H}$ as $L^{2}\left(R\left(q_{0}\right)\right)$, in the sense that for any $|\psi\rangle \in \mathcal{H}$ we have

$$
\begin{aligned}
\langle\psi \mid \psi\rangle & =\||\psi\rangle \|^{2}, \\
& =\int_{\mathcal{R}} \mathrm{d} q|\psi(q)|^{2}, \\
& =\int_{\mathcal{R}} \mathrm{d} p|\varphi(p)|^{2}, \\
& =\iint_{R\left(q_{0}\right)} \mathrm{d} q \mathrm{~d} p\left(|\chi(q, p)|^{2} \text { or }|\tilde{\chi}(q, p)|^{2}\right) .
\end{aligned}
$$

To recover all elements $|\psi\rangle \in \mathcal{H}$ we must allow for all (Lebesgue) square integrable Zak wavefunctions $\chi(q, p)$ or $\tilde{\chi}(q, p))$ over the phase space rectangle $R\left(q_{0}\right)$. The inverse of the Zak transform expresses $\psi(q)$ and $\varphi(p)$ in terms of $\chi\left(q^{\prime}, p^{\prime}\right)$ and $\chi\left(q^{\prime}, p^{\prime}\right)$ :

$$
\begin{gathered}
\psi(q)=\frac{q_{0}^{1 / 2}}{\sqrt{2 \pi}} \int_{-\pi / q_{0}}^{\pi / q_{0}} \mathrm{~d} p \mathrm{e}^{\mathrm{i} q p} \tilde{\chi}([q], p), \\
q=[q] \bmod q_{0}, \quad[q] \in\left(-\frac{1}{2} q_{0}, \frac{1}{2} q_{0}\right), \\
\varphi(p)=q_{0}^{-1 / 2} \int_{-\frac{1}{2} q_{0}}^{\frac{1}{2} q_{0}} \mathrm{~d} q \mathrm{e}^{-\mathrm{i} q p} \chi(q,[p]), \\
p=[p] \bmod 2 \pi / q_{0}, \quad[p] \in\left(-\pi / q_{0}, \pi / q_{0}\right) .
\end{gathered}
$$

The Zak basis vectors have the following formal 'periodicity' properties as are evident upon inspection from equations (2.13) and (2.14):

$$
\begin{gathered}
\left|q+q_{0}, p\right\rangle=\mathrm{e}^{-\mathrm{i} q_{0} p}|q, p\rangle, \\
\left|q, p+2 \pi / q_{0}\right\rangle=|q, p\rangle, \\
\left.\left.\mid q+q_{0}, p\right)=\mid q, p\right), \\
\left.\left.\mid q, p+2 \pi / q_{0}\right)=\mathrm{e}^{2 \pi \mathrm{i} q / q_{0}} \mid q, p\right) .
\end{gathered}
$$

These differing behaviours of $|q, p\rangle$ and $\mid q, p)$ are consistent with equation (2.16). Indeed the geometric phase factor appearing in equation (2.16) converts strict periodicity with respect to $p$ and periodicity upto a phase with respect to $q$ in the case of $|q, p\rangle$, to exactly opposite properties for $\mid q, p)$. The point to be now appreciated is that while for a general $|\psi\rangle \in \mathcal{H}$ we have no conditions on $\chi(q, p)$ (or $\tilde{\chi}(q, p)$ ) other than (Lebesgue) square integrability over $R\left(q_{0}\right)$, if we restrict ourselves to a subset of $|\psi\rangle \in \mathcal{H}$ possessing continuous Zak wavefunctions we can say something specific. Namely, based on equation (3.5) we have for such vectors in $\mathcal{H}$ the properties

$$
\begin{array}{r}
\chi\left(\frac{1}{2} q_{0}, p\right)=\mathrm{e}^{\mathrm{i} q_{0} p} \chi\left(-\frac{1}{2} q_{0}, p\right), \\
\chi\left(q, \pi / q_{0}\right)=\chi\left(q,-\pi / q_{0}\right), \\
\tilde{\chi}\left(\frac{1}{2} q_{0}, p\right)=\tilde{\chi}\left(-\frac{1}{2} q_{0}, p\right), \\
\tilde{\chi}\left(q, \pi / q_{0}\right)=\mathrm{e}^{-2 \pi \mathrm{i} q / q_{0}} \tilde{\chi}\left(q,-\pi / q_{0}\right) .
\end{array}
$$

For such vectors $|\psi\rangle \in \mathcal{H}$ these relations among the values of the Zak wavefunctions along the edges of $R\left(q_{0}\right)$ can be exploited to show that $\chi(q, p)$ (or $\tilde{\chi}(q, p))$ must have at least one zero in their domain of definition (see, for instance, Janssen [8], for a proof).

It is worth remarking that on account of the robustness of geometric phases, namely the impossibility of transforming them away by using phase redefinitions permitted by quantum mechanics, we cannot replace equations (3.6) in any natural way by some related wavefunctions over $R\left(q_{0}\right)$ strictly periodic simultaneously in both $q$ and $p$. At best the geometric phase $\mathrm{e}^{\mathrm{i} q p}$ of equation (2.16) can be shifted from one place to another; and its presence is the essential reason behind the interesting result mentioned in the previous paragraph.

It may be of interest to see briefly how the original operators $\hat{q}, \hat{p}$ obeying the commutation relation (2.1) act on the Zak wavefunctions. It turns out that in both cases we have to restrict the wavefunctions $\chi, \tilde{\chi}$ to be continuous and once differentiable in each argument (so that the periodicity conditions (3.6a) do apply) and then we have:

$$
\begin{gathered}
\chi(q, p): \hat{q}=q+\mathrm{i} \frac{\partial}{\partial p}, \quad \hat{p}=-\mathrm{i} \frac{\partial}{\partial q}, \\
\tilde{\chi}(q, p): \hat{q}=\mathrm{i} \frac{\partial}{\partial p}, \quad \hat{p}=p-\mathrm{i} \frac{\partial}{\partial q} .
\end{gathered}
$$

After this brief recollection of the Zak representation of quantum mechanics, we turn to the possibility of viewing the Zak basis states as an (ideal) system of generalized coherent states with respect to the $\mathrm{H}-\mathrm{W}$ group. At $q=p=0$ the connection (2.16) simplifies and we are led to define

$$
\left.\Psi_{0}=|0,0\rangle=\mid 0,0\right),
$$

it being understood that this is not a normalizable vector in $\mathcal{H}$. Now equations (2.13) and (2.14) show us how to build $|q, p\rangle$ and $\mid q, p)$ from $\Psi_{0}$ in natural ways using the displacement operators in equation (2.3):

$$
\begin{aligned}
|q, p\rangle & =\frac{q_{0}^{1 / 2}}{\sqrt{2 \pi}} \sum_{n \in \mathcal{Z}} \mathrm{e}^{\mathrm{i} n q_{0} p}\left|q+n q_{0}\right\rangle \\
& =V(q) \frac{q_{0}^{1 / 2}}{\sqrt{2 \pi}} \sum_{n \in \mathcal{Z}} \mathrm{e}^{\mathrm{i} i q_{0} p}\left|n q_{0}\right\rangle \\
& =V(q) U(p) \Psi_{0} \\
& =\mathrm{e}^{-\mathrm{i} q p / 2} D(q, p) \Psi_{0},
\end{aligned}
$$




$$
\begin{aligned}
\mid q, p) & \left.=q_{0}^{-1 / 2} \sum_{n \in \mathcal{Z}} \mathrm{e}^{-2 \pi \mathrm{i} n q / q_{0}} \mid p+2 \pi n / q_{0}\right) \\
& \left.=q_{0}^{-1 / 2} U(p) \sum_{n \in \mathcal{Z}} \mathrm{e}^{-2 \pi \mathrm{i} n q / q_{0}} \mid 2 \pi n / q_{0}\right) \\
& =U(p) V(q) \Psi_{0} \\
& =\mathrm{e}^{\mathrm{i} q p / 2} D(q, p) \Psi_{0} .
\end{aligned}
$$

This shows that the simultaneous eigenvectors of $U_{0}$ and $V_{0}$ actually form the orbit of the (ideal) fiducial vector $\Psi_{0}$ under the H-W group (save for the phase factor in $D\left(\alpha_{1}, \alpha_{2}, \alpha_{3}\right)$ ). Incidentally equations (3.9) show explicitly that the phases $\mathrm{e}^{\mathrm{i} q p}$ appearing in equations (2.6) and (2.16) have a common origin.

To identify the Zak basis vectors as a family of generalized coherent states, at least in a formal sense, we must identify within the $\mathrm{H}-\mathrm{W}$ group the stability group of the fiducial vector $\Psi_{0}$. From equations (2.13), (2.14) and (3.8), we have the obvious properties

$$
U_{0} \Psi_{0}=V_{0} \Psi_{0}=\Psi_{0},
$$

which lead to the invariances of the Zak basis states in the form

$$
\begin{gathered}
D\left(q \pm q_{0}, p\right) \Psi_{0}=\mathrm{e}^{\mp \mathrm{i} q_{0} p / 2} D(q, p) \Psi_{0}, \\
D\left(q, p \pm 2 \pi / q_{0}\right) \Psi_{0}=\mathrm{e}^{ \pm \mathrm{i} \pi q / q_{0}} D(q, p) \Psi_{0} .
\end{gathered}
$$

Thus the stability subgroup of $\Psi_{0}$ within the H-W group is an infinite discrete abelian subgroup $H_{q_{0}}$ generated by $U_{0}$ and $V_{0}$ :

$$
\begin{aligned}
H_{q_{0}} & =\left\{\mathrm{e}^{-\mathrm{i} \alpha_{3}+\mathrm{i} \alpha_{2} \hat{q}-\mathrm{i} \alpha_{1} \hat{p}} \mid \alpha_{3}=0, \alpha_{1}=n q_{0},\right. \\
\alpha_{2} & \left.=2 \pi m / q_{0}, m, n \in \mathcal{Z}\right\}=\left\{U_{0}^{m} V_{0}^{n} \mid m, n \in \mathcal{Z}\right\} .
\end{aligned}
$$

This means that the orbit of $\Psi_{0}$, namely the collection of states $\{|q, p\rangle\}$ say, is essentially the coset space of the H-W group with respect to $H_{q_{0}}$. This is identifiable with the rectangle $R\left(q_{0}\right)$ of area $2 \pi$ in phase space, and so we see again in a natural way why we may limit $(q, p)$ to this rectangle in the Zak representation.

To sum up, the simultaneous (ideal) eigenvectors of $U_{0}$ and $V_{0}$ form a system of generalized coherent states for the $\mathrm{H}-\mathrm{W}$ group, based on the fiducial vector $\Psi_{0}$ and identifiable with the coset space (H-W group) $/ H_{q_{0}}$. We must however note the following: unlike the usual cases of generalized coherent state systems arising from a fiducial vector which is a normalizable vector in $\mathcal{H}$, in which case the inner product of two generalized coherent states is generally nonzero (see the discussion in Klauder and Skagerstam in [5]), here we have

$$
\left(D\left(q^{\prime}, p^{\prime}\right) \Psi_{0}, D(q, p) \Psi_{0}\right)=\delta\left(q^{\prime}-q\right) \delta\left(p^{\prime}-p\right) .
$$

We realize that this result of orthonormality in the continuous Dirac sense is possible only because $\Psi_{0}$ is nonnormalizable.

\section{Wigner distribution for Zak fiducial vector}

The important role played by the fiducial vector $\Psi_{0}$ motivates us to explore its invariances in the Wigner representation language, more particularly since the primitive invariances are with respect to phase space displacements. The position and momentum space wavefunctions of $\Psi_{0}$ are:

$$
\begin{aligned}
& \Psi_{0}(q)=\left\langle q \mid \Psi_{0}\right\rangle=\frac{q_{0}^{1 / 2}}{\sqrt{2 \pi}} \sum_{n \in \mathcal{Z}} \delta\left(q-n q_{0}\right), \\
& \Phi_{0}(p)=\left(p\left|\Psi_{0}\right\rangle=q_{0}^{-1 / 2} \sum_{n \in \mathcal{Z}} \delta\left(p-2 \pi n / q_{0}\right), \quad q, p \in \mathcal{R} .\right.
\end{aligned}
$$

Notice that in both cases we have a periodic sequence of delta functions with uniform positive weights. Each of these is quite easily seen to display the basic invariances (3.10) of $\Psi_{0}$. From here we obtain the Wigner function corresponding to $\Psi_{0}$ :

$$
\begin{aligned}
& W_{0}(q, p)=\frac{1}{2 \pi} \int_{-\infty}^{\infty} \mathrm{d} q^{\prime} \Psi_{0}\left(q-\frac{1}{2} q^{\prime}\right) \Psi_{0}\left(q+\frac{1}{2} q^{\prime}\right)^{*} \mathrm{e}^{\mathrm{i} q^{\prime} p} \\
&=\frac{q_{0}}{(2 \pi)^{2}} \sum_{n, n^{\prime} \in \mathcal{Z}} \int_{-\infty}^{\infty} \mathrm{d} q^{\prime} \delta\left(q-\frac{1}{2} q^{\prime}-n q_{0}\right) \\
& \times \delta\left(q+\frac{1}{2} q^{\prime}-n^{\prime} q_{0}\right) \mathrm{e}^{\mathrm{i} q^{\prime} p} \\
&= \frac{q_{0}}{(2 \pi)^{2}} \sum_{n, n^{\prime} \in \mathcal{Z}} \delta\left(2 q-\left(n+n^{\prime}\right) q_{0}\right) \int_{-\infty}^{\infty} \mathrm{d} q^{\prime} \mathrm{e}^{\mathrm{i} q^{\prime} p} \\
& \times \delta\left(q-\frac{1}{2} q^{\prime}-n q_{0}\right), \\
&= \frac{q_{0}}{(2 \pi)^{2}} \sum_{n, n^{\prime} \in \mathcal{Z}} \delta\left(q-\frac{1}{2}\left(n+n^{\prime}\right) q_{0}\right) \mathrm{e}^{2 \mathrm{i} p\left(q-n q_{0}\right)} \\
&= \frac{q_{0}}{(2 \pi)^{2}} \mathrm{e}^{2 \mathrm{i} q p} \sum_{m, n \in \mathcal{Z}} \delta\left(q-\frac{1}{2} m q_{0}\right) \mathrm{e}^{-2 \mathrm{i} n p q_{0}} \\
&= \frac{q_{0}}{(2 \pi)^{2}} \mathrm{e}^{2 \mathrm{i} q p} \sum_{m \in \mathcal{Z}} \delta\left(q-\frac{m}{2} q_{0}\right) 2 \pi \frac{1}{2 q_{0}} \cdot \sum_{n \in \mathcal{Z}} \\
& \times \delta\left(p-\pi n / q_{0}\right) \\
&= \frac{1}{4 \pi} \sum_{m, n \in \mathcal{Z}}(-1)^{m n} \delta\left(q-\frac{m}{2} q_{0}\right) \delta\left(p-n \pi / q_{0}\right)
\end{aligned}
$$

We have here a lattice of delta functions in the $q-p$ phase plane, at the points $\left((m / 2) q_{0}, n \pi / q_{0}\right)$ for all $m, n \in \mathcal{Z}$. Thus the lattice spacings are $(1 / 2) q_{0}$ along the $q$-axis and $\pi / q_{0}$ along the $p$-axis. The primitive cell here is one-fourth of $R\left(q_{0}\right)$ encountered earlier in constructing the $U_{0}-V_{0}$ eigenstates. When $m$ and $n$ are both odd we have weight -1 , otherwise always weight +1 . This makes the invariances

$$
W_{0}\left(q \pm q_{0}, p\right)=W_{0}\left(q, p \pm 2 \pi / q_{0}\right)=W_{0}(q, p)
$$

immediately obvious. It is interesting to note that this (idealized) Wigner function and its properties are reminiscent of the Talbot grating in classical wave optics.

\section{The ST for band-limited wavefunctions}

The results so far discussed have depended on one positive parameter $q_{0}$ with dimension of length. We now turn to results which depend in addition on a second (positive) parameter $p_{0}$ with dimension of momentum, such that $p_{0} \leqslant 2 \pi / q_{0}$. The first is the ST recalled in this section [1]. The second, taken up in the next section, is the property of (over) completeness possessed by certain lattices of standard quantum mechanical (Schrödinger) coherent states. 
Let $|\psi\rangle \in \mathcal{H}$ be such that its momentum space wavefunction $\varphi(p)$ vanishes for $p$ outside the interval $\left[-\frac{1}{2} p_{0}, \frac{1}{2} p_{0}\right]$ of width $p_{0}$, for some positive $p_{0}$. Therefore,

$$
\psi(q)=\frac{1}{\sqrt{2 \pi}} \int_{-\frac{1}{2} p_{0}}^{\frac{1}{2} p_{0}} \mathrm{~d} p \mathrm{e}^{\mathrm{i} p q} \varphi(p) .
$$

We will then say that $\psi$ is band-limited and has bandwidth $p_{0}$. (Conventionally, the bandwidth is the length of the smallest closed interval, centred about zero, outside of which $\varphi(p)$ vanishes; however, in the present context it is more convenient to use the above definition, without insisting that $\varphi(p)$ be nonzero throughout the interval $\left[-\frac{1}{2} p_{0}, \frac{1}{2} p_{0}\right]$.) It is then useful to define a subspace $\mathcal{H}_{0}\left(p_{0}\right) \subset \mathcal{H}$, made up of bandlimited $\psi(q)$ with bandwidth $p_{0}$, as follows:

$$
\mathcal{H}_{0}\left(p_{0}\right)=\left\{|\psi\rangle \in \mathcal{H} \mid \varphi(p)=0 \quad \text { for } \quad p \notin\left[-\frac{1}{2} p_{0}, \frac{1}{2} p_{0}\right]\right\} \subset \mathcal{H}
$$

(The reason for the subscript zero will become clear in the following section.) We can now see that if $p_{0} \leqslant 2 \pi / q_{0}$, the subspace $\mathcal{H}_{0}\left(p_{0}\right)$ is simply characterized in terms of Zak wavefunctions, namely as is clear from equations (3.2) and (3.4):

$$
\begin{gathered}
|\psi\rangle \in \mathcal{H}_{0}\left(p_{0}\right), \\
p_{0} \leqslant 2 \pi / q_{0} \Leftrightarrow \quad \tilde{\chi}(q, p)=\tilde{\chi}(p) \text { independent of } q, \\
\varphi(p)=q_{0}^{1 / 2} \tilde{\chi}(p), \quad p \in\left[-\frac{1}{2} p_{0}, \frac{1}{2} p_{0}\right] \subseteq\left[-\pi / q_{0}, \pi / q_{0}\right] .
\end{gathered}
$$

We will hereafter regard $q_{0}$ as given right at the start and kept fixed, so that the domain of definition of Zak wavefunctions $\chi(q, p), \tilde{\chi}(q, p)$ is the rectangle $R\left(q_{0}\right)$ in phase space, and this is unvarying. The second parameter $p_{0}$ will be permitted to vary subject always to $p_{0} \leqslant 2 \pi / q_{0}$. With this understood, the relation (5.3) discloses a natural connection between position-independent Zak wavefunctions $\tilde{\chi}(q, p)$ and bandlimited wavefunctions $\psi(q)$ with bandwidth $p_{0}$.

Now we proceed to the ST. We appeal to the Poisson Summation Formula (2.19) which holds for any $q_{0}>0$. For a given band-limited $\psi(q)$ with bandwidth $p_{0} \leqslant 2 \pi / q_{0}$, i.e. $|\psi\rangle \in \mathcal{H}_{0}\left(p_{0}\right)$, the interval $\left[-\frac{1}{2} p_{0}, \frac{1}{2} p_{0}\right]$ does not extend beyond the interval $\left[-\pi / q_{0}, \pi / q_{0}\right]$. If we now take $p \in$ $\left[-\frac{1}{2} p_{0}, \frac{1}{2} p_{0}\right]$, all the conditions for the validity of equation (2.19) are obeyed and furthermore only the term $n=0$ survives on the right-hand side of that equation. Therefore, for $|\psi\rangle \in \mathcal{H}_{0}\left(p_{0}\right), p_{0} \leqslant 2 \pi / q_{0}$, we have:

$$
\begin{gathered}
\varphi(p)=\frac{q_{0}}{\sqrt{2 \pi}} \mathrm{e}^{-\mathrm{i} q^{\prime} p} \sum_{n \in \mathcal{Z}} \mathrm{e}^{-\mathrm{i} n q_{0} p} \psi\left(q^{\prime}+n q_{0}\right), \\
q^{\prime} \in\left[-\frac{1}{2} q_{0}, \frac{1}{2} q_{0}\right], \quad p \in\left[-\frac{1}{2} p_{0}, \frac{1}{2} p_{0}\right] \subseteq\left[-\pi / q_{0}, \pi / q_{0}\right] .
\end{gathered}
$$

Using this in equation (2.18) we are able to express $\psi(q)$ for any $q \in \mathcal{R}$ in terms of the discrete equispaced sequence of values $\psi\left(q^{\prime}+n q_{0}\right)$ :

$$
\begin{aligned}
\psi(q)= & \frac{q_{0}}{2 \pi} \sum_{n \in \mathcal{Z}} \psi\left(q^{\prime}+n q_{0}\right) \int_{-\frac{1}{2} p_{0}}^{\frac{1}{2} p_{0}} \mathrm{~d} p \mathrm{e}^{\mathrm{i} p\left(q-q^{\prime}-n q_{0}\right)} \\
= & \frac{q_{0}}{\pi} \sum_{n \in \mathcal{Z}} \frac{\sin \left\{p_{0}\left(q-q^{\prime}-n q_{0}\right) / 2\right\}}{\left(q-q^{\prime}-n q_{0}\right)} \psi\left(q^{\prime}+n q_{0}\right), \\
& q \in \mathcal{R}, \quad q^{\prime} \in\left[-\frac{1}{2} q_{0}, \frac{1}{2} q_{0}\right], \quad p_{0} \leqslant 2 \pi / q_{0} .
\end{aligned}
$$

This is, as is well known, the ST for band-limited $\psi(q)$. However, in the usual statement, the bandwidth $p_{0}$ is supposed to be known, and the inequality $p_{0} \leqslant 2 \pi / q_{0}$ is read as $q_{0} \leqslant$ $2 \pi / p_{0}$ and taken to mean that the values of $\psi\left(q^{\prime}+n q_{0}\right)$ are needed at sufficiently close spacing in order to be able to determine $\psi(q)$ for all $q$.

If in equation (5.5) we let $q \rightarrow q^{\prime}+m q_{0}$ for some $m \in \mathcal{Z}$, we find:

$$
\begin{aligned}
\psi\left(q^{\prime}+m q_{0}\right)= & \frac{q_{0}}{\pi}\left(\frac{p_{0}}{2} \psi\left(q^{\prime}+m q_{0}\right)\right. \\
& \left.+\sum_{\substack{n \in \mathcal{Z} \\
n \neq m}} \frac{\sin \left\{(m-n) q_{0} p_{0} / 2\right\}}{(m-n) q_{0}} \psi\left(q^{\prime}+n q_{0}\right)\right) \\
& q^{\prime} \in\left[-\frac{1}{2} q_{0}, \frac{1}{2} q_{0}\right] .
\end{aligned}
$$

For $p_{0}<2 \pi / q_{0}$ this shows that the values of $\psi(q)$ at the discrete set of points $q^{\prime}+n q_{0}$, while certainly adequate to determine $\psi(q)$ in its entirety, cannot be chosen independently. There are linear relations among them, and more such relations will be described below. For $p_{0}=2 \pi / q_{0}$, equation (5.6) becomes an identity.

In the form (5.5) for the ST, when $p_{0}<2 \pi / q_{0}$, the bandwidth $p_{0}$ appears explicitly on the right-hand side. It is interesting that there is an alternative derivation and expression of the ST, based on Cauchy's theorem for analytic functions, in which $p_{0}$ does not appear explicitly but only implicitly. From equation (5.1) it is evident that $\psi(q)$ is the boundary value, on the real axis, of an entire analytic function $\psi(z)$ defined for all $z \in \mathcal{C}$ by

$$
\psi(z)=\frac{1}{\sqrt{2 \pi}} \int_{-\frac{1}{2} p_{0}}^{\frac{1}{2} p_{0}} \mathrm{~d} p \mathrm{e}^{\mathrm{i} p z} \varphi(p)
$$

Whereas, by the Riemann-Lebesgue lemma, as $q \rightarrow \pm \infty$ along the real axis $\psi(q)$ definitely tends to zero, we now see from the band limitedness that as $|z| \rightarrow \infty$ in the complex plane the behaviour of $\psi(z)$ is controlled by

$$
|\psi(z)| \leqslant \text { constant } \exp \left(\frac{1}{2} p_{0}|\operatorname{Im} z|\right)
$$

Now, for fixed $q^{\prime} \in\left[-\frac{1}{2} q_{0}, \frac{1}{2} q_{0}\right]$, set up the analytic function

$$
f(z)=\frac{\pi}{\sin \pi z / q_{0}} \frac{\psi\left(q^{\prime}+z\right)}{q^{\prime}+z-z_{0}}
$$

where $z_{0} \in \mathcal{C}$ with $\operatorname{Im} z_{0} \neq 0$. This function has simple poles at $z=z_{0}-q^{\prime}$ and $z=n q_{0}, n \in \mathcal{Z}$. As $|z| \rightarrow \infty$, on account of (5.8) $|f(z)|$ tends to zero exponentially rapidly (and for this we do need the strict inequality $p_{0}<2 \pi / q_{0}$ ). Thus using Cauchy's residue theorem for a contour consisting of a circle of large radius centred at the origin, and letting the radius tend to infinity, we get the result

$$
\psi\left(z_{0}\right)=\frac{q_{0}}{\pi} \sin \left\{\pi\left(z_{0}-q^{\prime}\right) / q_{0}\right\} \sum_{n \in \mathcal{Z}}(-1)^{n} \frac{\psi\left(q^{\prime}+n q_{0}\right)}{\left(z_{0}-q^{\prime}-n q_{0}\right)} .
$$


We now let $z_{0} \rightarrow q \in \mathcal{R}$ to finally get:

$$
\begin{gathered}
\psi(q)=\frac{q_{0}}{\pi} \sin \left\{\pi\left(q-q^{\prime}\right) / q_{0}\right\} \sum_{n \in \mathcal{Z}}(-1)^{n} \frac{\psi\left(q^{\prime}+n q_{0}\right)}{\left(q-q^{\prime}-n q_{0}\right)}, \\
q^{\prime} \in\left[-\frac{1}{2} q_{0}, \frac{1}{2} q_{0}\right], \quad p_{0}<2 \pi / q_{0} .
\end{gathered}
$$

This differs in structure and properties from equation (5.5). As mentioned earlier, the bandwidth $p_{0}$ is not explicitly present on the right-hand side; and as $q \rightarrow q^{\prime}+m q_{0}$ for some $m \in \mathcal{Z}$, we get an identity rather than a nontrivial relation like (5.6). The fact that the values of $\psi\left(q^{\prime}+n q_{0}\right), n \in \mathcal{Z}$, are not all independent when $p_{0}<2 \pi / q_{0}$ permits the existence of both equations (5.5) and (5.11) having somewhat different forms. It is interesting to notice that even though we assumed $p_{0}<$ $2 \pi / q_{0}$ in the Cauchy theorem derivation of equation (5.11), if we do take $p_{0}=2 \pi / q_{0}$ the two results (5.5) and (5.11) become identical.

To show even more forcefully, when $p_{0}<2 \pi / q_{0}$, that $\psi\left(q^{\prime}+n q_{0}\right)$ for $n \in \mathcal{Z}$ are not all independent, consider in place of $f(z)$ of equation (5.9) the analytic function

$$
g(z)=\frac{\pi}{\sin \pi z / q_{0}} \cdot \psi\left(q^{\prime}+z\right) P(z),
$$

where $P(z)$ is any finite degree polynomial. The conditions for the use of Cauchy's theorem for the same circular contour as before, and going to the limit of infinite radius, are all obeyed. In that limit we get the result

$$
\sum_{n \in \mathcal{Z}}(-1)^{n} P\left(n q_{0}\right) \psi\left(q^{\prime}+n q_{0}\right)=0
$$

Thus we have infinitely many such linear dependence relations, the independent ones among them corresponding to choosing $P(z)$ to be any monomial $z^{m}, m \in \mathcal{Z}$. The important point is that in the above argument $P(z)$ must be a polynomial of finite degree. If it were a nontrivial entire function, its behaviour as $|z| \rightarrow \infty$ could spoil the behaviour of $g(z)$ and then Cauchy's theorem becomes inapplicable in general.

\section{Extended ST and standard coherent state lattices}

We have mentioned in section 1 that certain well-known theorems pertaining to phase space lattices of the standard coherent states in quantum mechanics have a character very similar to the ST discussed in the preceding section. Furthermore, the Zak representation of quantum mechanical wavefunctions has proven very useful in understanding (at least) the von Neumann lattice of standard coherent states, and in posing the problem of generalizing this lattice [8]. In this section, we combine the usual statement of the ST with the operator machinery provided by the H-W group to find the maximum extent to which the ST can be generalized and expressed in terms of the standard coherent states. Thus our aim is to see if the ST can be extended from vectors $|\psi\rangle \in \mathcal{H}_{0}\left(p_{0}\right)$ to all $|\psi\rangle \in \mathcal{H}$. We then state the known results about lattices of standard coherent states, and show how close the two results are in appearance and exactly where they differ.

We first recall briefly the definition and wavefunctions of the standard coherent states [2, 4], the actions of the phase space displacement operators on them, and an interesting way in which certain coherent states can be obtained from the (ideal) position and momentum eigenvectors $|q\rangle$ and $\mid p$ ). With this preparation we are able to recast and extend the ST in the language of phase space lattices of coherent states.

The standard coherent states are labelled by complex numbers $z \in \mathcal{C}$; for clarity they will be written as $\mid z)$ ). Their definition in terms of the $\mathrm{H}-\mathrm{W}$ displacement operators and their wavefunctions are:

$$
\begin{aligned}
z & =\frac{1}{\sqrt{2}}(q+\mathrm{i} p): \\
\mid z)) & \left.\left.=\mid \frac{1}{\sqrt{2}}(q+\mathrm{i} p)\right)\right) \\
& =D(q, p) \mid 0)) \\
& \left.\left.=e^{(\mathrm{i} / 2) q p} V(q) U(p) \mid 0\right)\right) \\
& \left.\left.=e^{-(\mathrm{i} / 2) q p} U(p) V(q) \mid 0\right)\right) \\
\left.\left.\left\langle q^{\prime}\right| z\right)\right)=\frac{1}{\pi^{1 / 4}} & \exp \left\{-\frac{\mathrm{i}}{2} q p+\mathrm{i} p q^{\prime}-\frac{1}{2}\left(q^{\prime}-q\right)^{2}\right\}, \\
\left.\left(p^{\prime} \mid z\right)\right)=\frac{1}{\pi^{1 / 4}} & \exp \left\{\frac{\mathrm{i}}{2} q p-\mathrm{i} q p^{\prime}-\frac{1}{2}\left(p^{\prime}-p\right)^{2}\right\} .
\end{aligned}
$$

These states are normalized to unity and no two of them are mutually orthogonal. They are (right) eigenstates of the annihilation operator $\hat{a}$ :

$$
\left.\left.\left.\left.\hat{a}=\frac{1}{\sqrt{2}}(\hat{q}+\mathrm{i} \hat{p}): \hat{a} \mid z\right)\right)=z \mid z\right)\right) .
$$

The actions of $V\left(q^{\prime}\right)$ and $U\left(p^{\prime}\right)$ are easily obtained:

$$
\begin{aligned}
& \left.\left.\left.\left.V\left(q^{\prime}\right) \mid \frac{1}{\sqrt{2}}(q+\mathrm{i} p)\right)\right)=\mathrm{e}^{-(\mathrm{i} / 2) \mathrm{pq}^{\prime}} \mid \frac{1}{\sqrt{2}}\left(\mathrm{q}+\mathrm{q}^{\prime}+\mathrm{ip}\right)\right)\right) \\
& \left.\left.\left.\left.U\left(p^{\prime}\right) \mid \frac{1}{\sqrt{2}}(q+\mathrm{i} p)\right)\right)=\mathrm{e}^{(\mathrm{i} / 2) q p^{\prime}} \mid \frac{1}{\sqrt{2}}\left(q+\mathrm{i} p+\mathrm{i} p^{\prime}\right)\right)\right) .
\end{aligned}
$$

It is interesting that particular cases of these coherent states can be obtained from the ideal vectors $|q\rangle$ and $\mid p$ ) by application of certain bounded hermitian operators to them [11]. Define two operators $S_{1}, S_{2}$ on $\mathcal{H}$ by

$$
S_{1}=\mathrm{e}^{-(1 / 2) \hat{q}^{2}}, \quad S_{2}=\mathrm{e}^{-(1 / 2) \hat{p}^{2}} .
$$

It is clear that they are both hermitian and bounded, while their inverses are hermitian and unbounded. Under similarity transformations applied respectively to $\hat{p}$ and to $\hat{q}$ we find:

$$
\begin{gathered}
S_{1} \hat{p} S_{1}^{-1}=-\mathrm{i} \sqrt{2} \hat{a}, \\
S_{2} \hat{q} S_{2}^{-1}=\sqrt{2} \hat{a} .
\end{gathered}
$$


Therefore $S_{1} \mid p$ ) and $S_{2}|q\rangle$ are particular coherent states $\left.\mid z\right)$ ). We find upon checking their wavefunctions that

$$
\begin{aligned}
& \left.\left.\left.S_{1} \mid p\right)=\frac{1}{\pi^{1 / 4} \sqrt{2}} \mid \frac{\mathrm{i}}{\sqrt{2}} p\right)\right), \\
& \left.\left.S_{2}|q\rangle=\frac{1}{\pi^{1 / 4} \sqrt{2}} \mid \frac{1}{\sqrt{2}} q\right)\right) .
\end{aligned}
$$

Hereafter, we mainly exploit equation (6.6b). On the basis of these relations we can express the content of the ST, equations (5.5) and (5.11), in an equivalent way in the language of these coherent states.

In equation (5.2) we have defined the subspace $\mathcal{H}_{0}\left(p_{0}\right) \subset$ $\mathcal{H}$ consisting of band-limited wavefunctions $\psi(q)$ with bandwidth $p_{0}$. Clearly $\mathcal{H}_{0}\left(p_{0}\right)$ is invariant under action by $S_{2}$, and moreover when restricted to $\mathcal{H}_{0}\left(p_{0}\right)$ the inverse $S_{2}^{-1}$ is also bounded. Now the content of the ST may be expressed in this way: given $q_{0}$ to begin with, ensuring $p_{0} \leqslant 2 \pi / q_{0}$ and choosing $q^{\prime} \in\left[-\frac{1}{2} q_{0}, \frac{1}{2} q_{0}\right]$,

$$
\begin{aligned}
& |\psi\rangle \in \mathcal{H}_{0}\left(p_{0}\right), \quad \psi\left(q^{\prime}+n q_{0}\right)=0, \\
& \text { all } n \in \mathcal{Z} \Rightarrow|\psi\rangle=0 .
\end{aligned}
$$

In other words such a band-limited $|\psi\rangle$ is (possibly over) determined by the values of $\psi\left(q^{\prime}+n q_{0}\right)$ for fixed $q^{\prime}$ and all $n \in \mathcal{Z}$. For simplicity now set $q^{\prime}=0$. Then the ST is equivalent to the statement

$$
\begin{aligned}
& |\psi\rangle \in \mathcal{H}_{0}\left(p_{0}\right), \quad\left\langle n q_{0} \mid \psi\right\rangle=0, \\
& \text { all } n \in \mathcal{Z} \Rightarrow|\psi\rangle=0 .
\end{aligned}
$$

The interesting aspect of this statement is that the (ideal) vectors $\left|n q_{0}\right\rangle$ are in no sense vectors in $\mathcal{H}_{0}\left(p_{0}\right)$, though they of course have nonzero projections on to $\mathcal{H}_{0}\left(p_{0}\right)$. Now from the above mentioned properties of $S_{2}$ with respect to $\mathcal{H}_{0}\left(p_{0}\right)$ we have on the one hand

$$
|\psi\rangle \in \mathcal{H}_{0}\left(p_{0}\right) \Longleftrightarrow S_{2}|\psi\rangle, S_{2}^{-1}|\psi\rangle \in \mathcal{H}_{0}\left(p_{0}\right) \quad,
$$

and on the other hand

$$
\begin{aligned}
& \left.\left.S_{2}\left|n q_{0}\right\rangle=\frac{1}{\pi^{1 / 4} \sqrt{2}} \mid \frac{1}{\sqrt{2}} n q_{0}\right)\right), \\
& \left\langle n q_{0}\right| S_{2}=\frac{1}{\pi^{1 / 4} \sqrt{2}}\left(\left(\frac{1}{\sqrt{2}} n q_{0} \mid .\right.\right.
\end{aligned}
$$

Combining these facts we see that the ST is equivalent to the following claim:

$$
\begin{aligned}
& |\psi\rangle \in \mathcal{H}_{0}\left(p_{0}\right), \quad\left(\left(\frac{1}{\sqrt{2}} n q_{0}|\psi\rangle=0,\right.\right. \\
& \text { all } n \in \mathcal{Z} \quad \Longrightarrow \quad|\psi\rangle=0 .
\end{aligned}
$$

This is so even though again $\left.\left.\mid \frac{1}{\sqrt{2}} n q_{0}\right)\right) \notin \mathcal{H}_{0}\left(p_{0}\right)$. Thus bandlimited $|\psi\rangle$ are (possibly over)determined by the overlaps $\left(\left(\frac{1}{\sqrt{2}} n q_{0}|\psi\rangle\right.\right.$ of $|\psi\rangle$ with a discrete sequence of (normalized!) coherent states, provided $p_{0} \leqslant 2 \pi / q_{0}$.

We can now see that in this form the ST permits an extension to all vectors in $\mathcal{H}$, using the properties (6.3) of the standard coherent states. We define a sequence of pairwise orthogonal subspaces $\mathcal{H}_{m}\left(p_{0}\right) \subset \mathcal{H}$ for all $m \in \mathcal{Z}$ by:

$$
\begin{aligned}
\mathcal{H}_{m}\left(p_{0}\right)= & \left\{| \psi \rangle \in \mathcal { H } | \varphi ( p ) = 0 \text { for } p \notin \left[\left(m-\frac{1}{2}\right) p_{0},\right.\right. \\
& \left.\left.\left(m+\frac{1}{2}\right) p_{0}\right]\right\} \subset \mathcal{H} \\
\mathcal{H}= & \sum_{m \in \mathcal{Z}} \mathcal{H}_{m}\left(p_{0}\right)
\end{aligned}
$$

(Now the meaning of the subscript in $\mathcal{H}_{m}\left(p_{0}\right)$ is evident.) Thus $\mathcal{H}$ consists of all off-centre band-limited wavefunctions $\psi(q)$ such that the centre of the momentum space interval is shifted from zero to $m p_{0}$, the width remaining $p_{0}$. On the one hand one sees easily that the $\mathcal{H}_{m}\left(p_{0}\right)$ arise from $\mathcal{H}_{0}\left(p_{0}\right)$ by action by integer powers of the momentum space displacement operator $U\left(p_{0}\right)$ :

$$
\begin{aligned}
\mathcal{H}_{m}\left(p_{0}\right) & =U\left(p_{0}\right)^{m} \mathcal{H}_{0}\left(p_{0}\right) \\
& =U\left(m p_{0}\right) \mathcal{H}_{0}\left(p_{0}\right), \quad m \in \mathcal{Z},
\end{aligned}
$$

and on the other hand each $\mathcal{H}_{m}\left(p_{0}\right)$ is invariant under action by $S_{2}$ as well as by $S_{2}^{-1}$. Moreover, when restricted to any $\mathcal{H}_{m}\left(p_{0}\right)$ (or any direct sum of them over a finite range of $m$ values), both these operators remain bounded. It is also clear that under the action by $U\left(m p_{0}\right)$, we have the twin results:

$$
\begin{gathered}
\left.\left.\left.\left.U\left(m p_{0}\right) \mid \frac{1}{\sqrt{2}} n q_{0}\right)\right)=\mid \frac{1}{\sqrt{2}}\left(n q_{0}+\mathrm{i} m p_{0}\right)\right)\right), \\
\left(\left(\frac{1}{\sqrt{2}} n q_{0} \mid U\left(m p_{0}\right)^{-1}=\left(\left(\frac{1}{\sqrt{2}}\left(n q_{0}+\mathrm{i} m p_{0}\right) \mid,\right.\right.\right.\right. \\
|\psi\rangle \in \mathcal{H}_{0}\left(p_{0}\right) \Longleftrightarrow U\left(m p_{0}\right)|\psi\rangle \in \mathcal{H}_{m}\left(p_{0}\right) .
\end{gathered}
$$

We can now transfer the statement (6.11) of the ST from $\mathcal{H}_{0}\left(p_{0}\right)$ to each $\mathcal{H}_{m}\left(p_{0}\right)$ individually:

$$
|\psi\rangle \in \mathcal{H}_{m}\left(p_{0}\right), \quad\left(\left(\frac{1}{\sqrt{2}}\left(n q_{0}+\mathrm{i} m p_{0}\right) \mid \psi\right)=0,\right.
$$

$$
\text { all } n \in \mathcal{Z} \quad \Longrightarrow \quad|\psi\rangle=0 \text {. }
$$

In other words such a band-limited $|\psi\rangle$ is (possibly over) determined by its inner products with the standard coherent states $\left.\left.\mid 1 / \sqrt{2}\left(n q_{0}+\mathrm{i} m p_{0}\right)\right)\right)$ keeping $m$ fixed and taking all $n \in \mathcal{Z}$. Once again we appreciate that this is so even though these coherent states are not in $\mathcal{H}_{m}\left(p_{0}\right)$.

To pass from $\mathcal{H}_{m}\left(p_{0}\right)$ to $\mathcal{H}$ is quite easy. We define the projection operators $P_{m}\left(p_{0}\right)$ on to the various orthogonal subspaces $\mathcal{H}_{m}\left(p_{0}\right)$ with standard properties:

$$
\begin{aligned}
P_{m}\left(p_{0}\right) & \left.=\int_{\left(m-\frac{1}{2}\right) p_{0}}^{\left(m+\frac{1}{2}\right) p_{0}} \mathrm{~d} p \mid p\right)(p \mid \\
& =U\left(m p_{0}\right) P_{0}\left(p_{0}\right) U\left(m p_{0}\right)^{-1}, \\
P_{m^{\prime}}\left(p_{0}\right) P_{m}\left(p_{0}\right) & =\delta_{m^{\prime} m} P_{m}\left(p_{0}\right), \\
P_{m}\left(p_{0}\right) S_{2} & =S_{2} P_{m}\left(p_{0}\right) .
\end{aligned}
$$


Then the content of the original ST is fully equivalent to the following:

$$
\begin{aligned}
& |\psi\rangle \in \mathcal{H}, \quad\left(\left(\frac{1}{\sqrt{2}}\left(n q_{0}+\mathrm{i} m p_{0}\right)\left|P_{m}\left(p_{0}\right)\right| \psi\right\rangle=0,\right. \\
& \text { all } m, n \in \mathcal{Z} \Longrightarrow|\psi\rangle=0, \quad p_{0} \leqslant 2 \pi / q_{0} .
\end{aligned}
$$

It is worthwhile exploring a little bit the real meaning of implication statements such as equations (6.8), (6.11), (6.15) and (6.17) in the following manner. The subtleties mainly arise from the use of nonorthonormal systems of vectors as 'bases' in infinite dimensional Hilbert space. If one has a complete orthonormal basis $\left\{\left|e_{n}\right\rangle, n=1,2, \ldots\right\}$ for a Hilbert space $\mathcal{H}$, then any vector $|\psi\rangle \in \mathcal{H}$ has welldefined projections $\left\langle e_{n} \mid \psi\right\rangle$ on to these basis vectors, and the expansion of $|\psi\rangle$ in terms of $\left|e_{n}\right\rangle$ with these projections as coefficients indeed converges to $|\psi\rangle$ in norm. The inclusion of more and more terms in the expansion improves the accuracy with which $|\psi\rangle$ is approximated, while in the process the coefficients of already included terms suffer no change. Moreover, the vanishing of $\left\langle e_{n} \mid \psi\right\rangle$ for all $n$ implies the vanishing of $|\psi\rangle$. Lastly we can in principle choose each projection $\left\langle e_{n} \mid \psi\right\rangle$ independently as we wish, provided that the norm of $|\psi\rangle$ is kept finite.

If we now replace the orthonormal basis $\left\{\left|e_{n}\right\rangle\right\}$ by a nonorthonormal one, $\left\{\left|f_{n}\right\rangle\right\}$ say, which may in particular be overcomplete, the statements that can be made get modified. In general, the inner products $\left\langle f_{n} \mid \psi\right\rangle$ may not be specifiable independently of one another (over completeness of $\left\{\left|f_{n}\right\rangle\right\}$ ). On the other hand, the vanishing of all $\left\langle f_{n} \mid \psi\right\rangle$ indeed implies the vanishing of $|\psi\rangle$ (totality of $\left\{\left|f_{n}\right\rangle\right\}$ ). This means that the closure of the set of all finite linear combinations of the $\left|f_{n}\right\rangle$ is the total space $\mathcal{H}$. However, even given all these properties, there may be no definite set of expansion coefficients with whose help $|\psi\rangle$, in general, can be expressed as a convergent linear combination of the $\left|f_{n}\right\rangle$. (Over) completeness of $\left\{\left|f_{n}\right\rangle\right\}$ will ensure that any $|\psi\rangle$ can be approximated as closely as desired via finite linear combinations of the $\left|f_{n}\right\rangle$, but 'in the limit' there may be no 'actual expansion' for $|\psi\rangle$ in terms of $\left|f_{n}\right\rangle$. Vectors $|\psi\rangle$ in $\mathcal{H}$ expressible as finite linear combinations of the $\left|f_{n}\right\rangle$ or as infinite convergent linear combinations with well-defined expansion coefficients will form a dense subset in $\mathcal{H}$. This situation is well known in the theory of nonharmonic Fourier series [12]. It has also been analysed to a considerable extent in the case of the von Neumann lattice of standard coherent states, clarifying the meaning of expansions of vectors in terms of them or of their dual basis vectors [13].

Keeping all these subtleties in mind, let us agree to use the word 'basis' in a broad sense for a general possibly over complete set of possibly nonorthonormal vectors in $\mathcal{H}$. Then the final result of the original ST of equations (5.5), (5.11) and (6.11) is:

$$
\begin{aligned}
& \left.\left.\left\{P_{m}\left(p_{0}\right) \mid \frac{1}{\sqrt{2}}\left(n q_{0}+\mathrm{i} m p_{0}\right)\right)\right), n \in \mathcal{Z}, m \text { fixed }\right\} \\
& =\text { basis for } \mathcal{H}_{m}\left(p_{0}\right), \\
& \left.\left.\quad\left\{P_{m}\left(p_{0}\right) \mid \frac{1}{\sqrt{2}}\left(n q_{0}+\mathrm{i} m p_{0}\right)\right)\right), n, m \in \mathcal{Z}\right\} \\
& \quad=\text { basis for } \mathcal{H}, \quad p_{0} \leqslant 2 \pi / q_{0} .
\end{aligned}
$$

It has led to a basis for $\mathcal{H}$ by setting up bases for each $\mathcal{H}_{m}\left(p_{0}\right)$ in turn, and then taking the union over $m \in \mathcal{Z}$.

At this point we turn to the well-known results concerning lattices of standard coherent states, which have been mentioned earlier. These lattices consist of the vectors $\left.\left.\mid \frac{1}{\sqrt{2}}\left(n q_{0}+\mathrm{i} m p_{0}\right)\right)\right)$ with $n, m \in \mathcal{Z}$ and $p_{0} \leqslant 2 \pi / q_{0}$. For $p_{0}=$ $2 \pi / q_{0}$ we have the von Neumann lattice, while for $p_{0}<$ $2 \pi / q_{0}$ we have a finer lattice. Then we have the result [7]

$$
\begin{aligned}
&|\psi\rangle \in \mathcal{H}, \quad\left(\left(\frac{1}{\sqrt{2}}\left(n q_{0}+\mathrm{im} p_{0}\right)|\psi\rangle=0,\right.\right. \\
& \text { all } m, n \in \mathcal{Z} \Longrightarrow|\psi\rangle=0 .
\end{aligned}
$$

Thus the von Neumann (or any finer) lattice forms a basis for $\mathcal{H}$. At $p_{0}=2 \pi / q_{0}$ (von Neumann Case) we have over completeness by one vector, while for $p_{0}<2 \pi / q_{0}$ removal of any finite set of vectors from the lattice does not destroy over completeness. Of course for coarser lattices, $p_{0}>2 \pi / q_{0}$, totality is lost.

We can now appreciate how tantalizingly close the statements based on the ST and on the well-known quantum mechanical theory of coherent state lattices are to one another. The former leads to the twin statements (by virtue of symmetry between $\hat{q}, \hat{p}$ ):

$$
\begin{gathered}
\left.\left.\left\{P_{m}\left(p_{0}\right) \mid \frac{1}{\sqrt{2}}\left(n q_{0}+\mathrm{i} m p_{0}\right)\right)\right), m, n \in \mathcal{Z}\right\}=\text { basis for } \mathcal{H}, \\
\left.\left.\left\{\tilde{P}_{n}\left(q_{0}\right) \mid \frac{1}{\sqrt{2}}\left(n q_{0}+\mathrm{i} m p_{0}\right)\right)\right), m, n \in \mathcal{Z}\right\}=\text { basis for } \mathcal{H}, \\
q_{0} p_{0} \leqslant 2 \pi,
\end{gathered}
$$

where the new projection operators $\tilde{P}_{n}\left(q_{0}\right)$ are defined analogously to equation (6.16):

$$
\tilde{P}_{n}\left(q_{0}\right)=\int_{\left(n-\frac{1}{2}\right) q_{0}}^{\left(n+\frac{1}{2}\right) q_{0}} \mathrm{~d} q|q\rangle\langle q| .
$$

The latter leads to the statement

$\left.\left.\left\{\mid \frac{1}{\sqrt{2}}\left(n q_{0}+\mathrm{i} m p_{0}\right)\right)\right), m, n \in \mathcal{Z}\right\}=$ basis for $\mathcal{H}$.

These are two distinct properties possessed by the same lattices of standard coherent states. It may not be out of place to mention that all the results flowing from the ST are ultimately based on the properties of the Fourier transformation, while the results concerning von Neumann or finer standard coherent state lattices are generally derived by appealing to the sophisticated theory of entire analytic functions, and relations between their orders and types and distribution of zeros.

\section{The ST and lattice systems of H-W generalized coherent states}

We have seen how to express the ST in the language of standard coherent states, and how close the results are to earlier results pertaining to certain phase space lattices of the latter. Now, as mentioned in section 1 and as seen in section 3 in an idealized sense for the Zak basis vectors $|q, p\rangle$, the 
standard coherent states have been extended to systems of generalized coherent states (GCS) associated with the H-W group, obtained by replacing the Fock ground state $\mid 0)$ ) in equation (6.1) by a general normalized fiducial vector $\left|\psi_{0}\right\rangle \in$ $\mathcal{H}[4,7]$. Since the ST in itself does not refer to any coherent state system at all, it is natural to ask if its content could be expressed in terms of certain lattices of suitably chosen GCS systems as well. We shall find that this can sometimes be done. This section will explore the interrelations among H-W GCS systems, von Neumann type and finer lattices of such systems, the Zak representation and the ST. The new terms appearing here will be defined as we proceed. While for completeness some old results will be briefly recapitulated and sometimes sharpened, we will arrive at several new insights and results as well. As we shall throughout be concerned with the H-W group, continual reference to this group will be avoided.

Let $\left|\psi_{0}\right\rangle \in \mathcal{H}$ be a general normalized fiducial vector, with Schrödinger, momentum and Zak wavefunctions $\psi_{0}(q), \varphi_{0}(p), \chi_{0}(q, p)$ respectively. (Remember that the last of these depends on the parameter $q_{0}$.) The system of GCS based on $\left|\psi_{0}\right\rangle$, referred to as $\psi_{0}$-GCS hereafter, is defined as the family of normalized vectors

$$
\left|q^{\prime}, p^{\prime} ; \psi_{0}\right\rangle=D\left(q^{\prime}, p^{\prime}\right)\left|\psi_{0}\right\rangle, \quad\left(q^{\prime}, p^{\prime}\right) \in \mathcal{R}^{2} .
$$

It is a well-known result that for any choice of $\left|\psi_{0}\right\rangle$, the $\psi_{0^{-}}$ GCS family is total, i.e. (over) complete in $\mathcal{H}$ (Klauder and Skagerstam in [5] p 21). This is a consequence of the square integrable property of the unique UIR of the H-W group.

To obtain the Zak wavefunctions of the $\psi_{0}$-GCS, we need the effect of a general phase space displacement operator $D\left(q^{\prime}, p^{\prime}\right)$ on a Zak basis vector $|q, p\rangle$. From the results in sections 3 and 4 we find:

$$
\begin{aligned}
& (q, p) \in R\left(q_{0}\right), \quad\left(q^{\prime}, p^{\prime}\right) \in \mathcal{R}^{2}: \\
& D\left(q^{\prime}, p^{\prime}\right)|q, p\rangle=\mathrm{e}^{-\mathrm{i} \xi\left(q, p,-q^{\prime},-p^{\prime}\right)}\left|\left[q+q^{\prime}\right],\left[p+p^{\prime}\right]\right\rangle \\
& \langle q, p| D\left(q^{\prime}, p^{\prime}\right)=\mathrm{e}^{\mathrm{i} \xi\left(q, p, q^{\prime}, p^{\prime}\right)}\left\langle\left[q-q^{\prime}\right],\left[p-p^{\prime}\right]\right| \\
& \xi\left(q, p, q^{\prime}, p^{\prime}\right)= \\
& q p^{\prime}-p q^{\prime}+\frac{1}{2}\left(q p+q\left[p-p^{\prime}\right]-p\left[q-q^{\prime}\right]\right. \\
& \left.-\left[q-q^{\prime}\right]\left[p-p^{\prime}\right]\right)
\end{aligned}
$$

Here the fractional parts $\left[q \pm q^{\prime}\right],\left[p \pm p^{\prime}\right]$ are defined as in equation (3.4). We then find that the Zak wavefunctions of the vectors in the $\psi_{0}$-GCS are given in terms of $\chi_{0}$ by:

$\left\langle q, p \mid q^{\prime}, p^{\prime} ; \psi_{0}\right\rangle=\mathrm{e}^{\mathrm{i} \xi\left(q, p, q^{\prime}, p^{\prime}\right)} \chi_{0}\left(\left[q-q^{\prime}\right],\left[p-p^{\prime}\right]\right)$.

These are thus phase factors times phase space translations (reduced to or modulo $\left.R\left(q_{0}\right)\right)$ of $\chi_{0}(q, p)$.

The von Neumann lattice of $\psi_{0}$-GCS is the discrete ( $q_{0}$-dependent) subset of the states (7.1) defined as follows:

$$
\begin{aligned}
\left|n, m ; \psi_{0}\right\rangle & \equiv\left|n q_{0}, 2 \pi m / q_{0} ; \psi_{0}\right\rangle, \\
& =(-1)^{m n} U_{0}^{m} V_{0}^{n}\left|\psi_{0}\right\rangle, \quad n, m \in \mathcal{Z} .
\end{aligned}
$$

We shall refer to these as the $\psi_{0}$-von Neumann GCS lattice. Their Zak wavefunctions are naturally simpler than the general case in equation (7.3):

$\left\langle q, p \mid n, m ; \psi_{0}\right\rangle=(-1)^{m n} \mathrm{e}^{-\mathrm{i} n q_{0} p+2 \pi \mathrm{i} m q / q_{0}} \chi_{0}(q, p)$.
Naturally no translations of the arguments of $\chi_{0}$ are involved. Two noteworthy results which have been obtained very simply via the Zak description, may be recalled at this point (Bacry et al [8]):

$$
\begin{aligned}
& \left\{\left|n, m ; \psi_{0}\right\rangle\right\} \text { total in } \mathcal{H} \quad \Longleftrightarrow \quad \chi_{0}(q, p) \neq 0, \\
& \quad(q, p) \in R\left(q_{0}\right) ; \\
& \left\{\left|n, m ; \psi_{0}\right\rangle\right\} \text { orthonormal } \Longleftrightarrow\left|\chi_{0}(q, p)\right|=1, \\
& (q, p) \in R\left(q_{0}\right) .
\end{aligned}
$$

We see quite interestingly that property (7.6b) implies (7.6a): if the vectors of the $\psi_{0}$-von Neumann GCS lattice are mutually orthogonal, they are also complete in $\mathcal{H}$.

A connection to band-limited wavefunctions may now be easily seen. Suppose $\left|\psi_{0}\right\rangle \in \mathcal{H}_{0}\left(p_{0}\right)$ for some $p_{0}<2 \pi / q_{0}$, from equations (5.3) we know that then

$$
\chi_{0}(q, p)=q_{0}^{-1 / 2} \mathrm{e}^{\mathrm{i} q p} \varphi_{0}(p),
$$

and this certainly does not obey either equations in (7.6). Thus for such band-limited $\left|\psi_{0}\right\rangle$, even though the $\psi_{0}$-GCS is total, the $\psi_{0}$-von Neumann GCS lattice is neither orthonormal nor total.

Finer lattices of $\psi_{0}$-GCS than the von Neumann lattice are naturally defined in terms of a pair $\left(q_{0}, p_{0}\right)$ obeying $p_{0}<$ $2 \pi / q_{0}$. We shall simply call them $\psi_{0}$-finer GCS lattices and define their elements by:

$$
\begin{aligned}
\left|n q_{0}, m p_{0} ; \psi_{0}\right\rangle & =D\left(n q_{0}, m p_{0}\right)\left|\psi_{0}\right\rangle \\
& =\mathrm{e}^{\mathrm{i} m n q_{0} p_{0} / 2} U\left(m p_{0}\right) V_{0}^{n}\left|\psi_{0}\right\rangle \\
& =\mathrm{e}^{-\mathrm{i} m n q_{0} p_{0} / 2} V_{0}^{n} U\left(m p_{0}\right)\left|\psi_{0}\right\rangle, \quad n, m \in \mathcal{Z} .
\end{aligned}
$$

Since $U\left(m p_{0}\right)$ is not now an integer power of $U_{0}$, their Zak wavefunctions are not as simple as in equation (7.5). We shall see from the ST that we can derive some properties of totality for such finer lattices, analogous to the results of section 6 .

To proceed in this direction let us recall the way in which the ST was related to lattices of standard coherent states in section 6 . It was by realizing that the (ideal) position eigenket $|0\rangle$ and the Fock ground state $\mid 0)$ ) are related by the bounded invertible hermitian operator $S_{2}=\mathrm{e}^{-(1 / 2) \hat{p}}$ :

$$
\left.\mid 0))=\sqrt{2} \pi^{1 / 4} S_{2}|0\rangle=\frac{1}{\pi^{1 / 4}} \int_{-\infty}^{\infty} \mathrm{d} p \mathrm{e}^{-\frac{1}{2} p^{2}} \mid p\right) .
$$

As is evident, the momentum space wavefunction of 10$)$ ) is essentially $\mathrm{e}^{-(1 / 2) p^{2}}$ which is (i) square integrable, (ii) bounded and (iii) non-vanishing for all (finite) $p$. This gives us the hint to link up the ST to suitably chosen lattices of certain $\psi_{0}$-GCS systems.

Assume that the fiducial vector $\left|\psi_{0}\right\rangle$ has a momentum space wavefunction $\varphi_{0}(p)$ which is (of course) square integrable, bounded for all $p$ and non-vanishing for all (finite) $p$. It can in general be complex. Then we can express $\left|\psi_{0}\right\rangle$ in 
the following manner:

$$
\begin{aligned}
\left|\psi_{0}\right\rangle & \left.=\int_{-\infty}^{\infty} \mathrm{d} p \varphi_{0}(p) \mid p\right) \\
& \left.=S \int_{-\infty}^{\infty} \mathrm{d} p \mid p\right) \\
& =\sqrt{2 \pi} S|0\rangle \\
\left\langle\psi_{0}\right| & =\sqrt{2 \pi}\langle 0| S^{\dagger}, S=\varphi_{0}(\hat{p}) .
\end{aligned}
$$

The similarity to equation (7.9) is clear; however, $S$ unlike $S_{2}$ may not be hermitian. Now from the properties assumed for $\varphi_{0}(p)$ we see that both $S^{-1}$ and $S^{\dagger-1}$, while definable since $\varphi_{0}(p)$ is always nonzero, are expected to be unbounded since $\varphi_{0}(p) \rightarrow 0$ as $p \rightarrow \pm \infty$. However, upon restriction to the subspace $\mathcal{H}_{0}\left(p_{0}\right)$, all the four operators $S, S^{-1}, S^{\dagger}, S^{\dagger-1}$ are well defined and leave this subspace invariant. As in equation (6.9) here we have

$$
\begin{gathered}
|\psi\rangle \in \mathcal{H}_{0}\left(p_{0}\right) \Longleftrightarrow S|\psi\rangle, \quad S^{-1}|\psi\rangle, \quad S^{\dagger}|\psi\rangle, \\
S^{\dagger-1}|\psi\rangle \in \mathcal{H}_{0}\left(p_{0}\right) .
\end{gathered}
$$

Now we bring in the ST in the form (6.8) and combine it with equations (7.10) and (7.11). Subject to $p_{0} \leqslant 2 \pi / q_{0}$ and since $V_{0}$ commutes with $S$ and $S^{\dagger}$, it is equivalent to the statement

$$
\begin{gathered}
|\psi\rangle \in \mathcal{H}_{0}\left(p_{0}\right), \quad\left\langle\psi_{0}\left|V_{0}^{n}\right| \psi\right\rangle=0, \\
\text { all } n \in \mathcal{Z} \quad \Longrightarrow \quad|\psi\rangle=0 .
\end{gathered}
$$

The vectors $V_{0}^{n}\left|\psi_{0}\right\rangle$ are particular elements of the $\psi_{0}$-von Neumann or $\psi_{0}$-finer GCS lattice defined in equations (7.4) and (7.8) above:

$$
V_{0}^{n}\left|\psi_{0}\right\rangle=\left|n q_{0}, 0 ; \psi_{0}\right\rangle
$$

Therefore, we can reexpress the ST (7.12) as:

$$
\begin{gathered}
|\psi\rangle \in \mathcal{H}_{0}\left(p_{0}\right), \quad\left\langle n q_{0}, 0 ; \psi_{0} \mid \psi\right\rangle=0, \\
\text { all } n \in \mathcal{Z} \quad \Longrightarrow \quad|\psi\rangle=0 .
\end{gathered}
$$

This is a generalization of (6.11) valid (at least) when $\varphi_{0}(p)$ obeys the stated conditions. We see here too, as in section 6 , that even though the vectors $\left|n q_{0}, 0 ; \psi_{0}\right\rangle$ do not belong to $\mathcal{H}_{0}\left(p_{0}\right)$, the overlaps of a band-limited $|\psi\rangle \in \mathcal{H}_{0}\left(p_{0}\right)$ with them are enough to (possibly over) determine $|\psi\rangle$.

This result can next be extended to all the subspaces $\mathcal{H}_{m}\left(p_{0}\right)$ defined in equation (6.12). On the one hand we have equation (6.13) connecting $\mathcal{H}_{0}\left(p_{0}\right)$ to $\mathcal{H}_{m}\left(p_{0}\right)$. On the other hand we have from equation (7.8):

$$
\begin{aligned}
& \left|n q_{0}, m p_{0} ; \psi_{0}\right\rangle=\mathrm{e}^{\mathrm{i} m n q_{0} p_{0} / 2} U\left(m p_{0}\right)\left|n q_{0}, 0 ; \psi_{0}\right\rangle, \\
& \left\langle n q_{0}, m p_{0} ; \psi_{0}\right|=\mathrm{e}^{-\mathrm{i} m n q_{0} p_{0} / 2}\left\langle n q_{0}, 0 ; \psi_{0}\right| U\left(m p_{0}\right)^{-1} .
\end{aligned}
$$

Then combining equations (6.13) and (7.15) and the form (7.14) of the ST we arrive at the statement:

$$
\begin{gathered}
|\psi\rangle \in \mathcal{H}_{m}\left(p_{0}\right), \quad\left\langle n q_{0}, m p_{0} ; \psi_{0} \mid \psi\right\rangle=0, \\
\text { all } n \in \mathcal{Z} \Longrightarrow|\psi\rangle=0 .
\end{gathered}
$$

This generalizes equation (6.15) to those fiducial vectors $\left|\psi_{0}\right\rangle$ whose momentum space wavefunctions $\varphi_{0}(p)$ are pointwise non-vanishing and bounded. Bringing in the projection operators $P_{m}\left(p_{0}\right)$ on to $\mathcal{H}_{m}\left(p_{0}\right)$ defined in equation (6.16), we can give the extended form of the ST to von Neumann or finer GCS lattices in $\mathcal{H}$ :

$\varphi_{0}(p)$ normalizable, bounded, pointwise non-vanishing $\Longrightarrow$ $\left\{P_{m}\left(p_{0}\right)\left|n q_{0}, m p_{0} ; \psi_{0}\right\rangle, n, m \in \mathcal{Z}\right\}$ total in $\mathcal{H}, \quad p_{0} \leqslant 2 \pi / q_{0}$.

We can now summarize our findings. From the standpoint of the ST the 'best statement' in the direction of totality of suitable lattices of GCS is given by equation (7.17), and here the presence of the projections $P_{m}\left(p_{0}\right)$ is unavoidable as they reflect the band limitedness property basic to the $\mathrm{ST}$. This statement is available for both $p_{0}<2 \pi / q_{0}$ (finer lattices) and $p_{0}=2 \pi / q_{0}$ (von Neumann lattices). On the other hand, if we ask for the 'best statements' that can be made directly about totality of these lattices, independent of the ST and avoiding the projections $P_{m}\left(p_{0}\right)$, the picture is somewhat complicated. For $\psi_{0}$-von Neumann GCS lattices we have the result (7.6a) obtained most effectively by exploiting the Zak representation. For $\psi_{0}$-finer GCS lattices there seem to be no comparable general results, as the Zak representation cannot be easily exploited and we have no recourse to the theory of entire functions either.

To all this we must add the remark that boundedness and pointwise nonvanishing of $\varphi_{0}(p)$, and pointwise nonvanishing of $\chi_{0}(q, p)$, are properties not easily related to one another. In the case of the standard coherent states, studied in section 6 , both conditions happen to be satisfied; and for $p_{0}<2 \pi / q_{0}$ the theory of entire functions comes to our aid. These remarks suggest that there are two independent lines of argument at work here, leading to results of somewhat divergent characters.

\section{Concluding remarks}

In this work we have given an account of the interrelations between the Poisson Summation Formula and ST on the one hand and specific families of coherent state lattices associated with the H-W group on the other. In particular, by analysing the content of the usual ST from this perspective we are able to arrive at certain results on standard coherent state lattices which come pretty close to known results on von Neumann and finer standard coherent state lattices without recourse to the theory of entire analytic functions. We then pursue this line of thought further and show that it enables us to make specific statements concerning generalized coherent state lattice systems as well. We hope that the unified perspective developed here would evidently deepen our understanding of these matters and point the way to further interesting developments and generalizations. 


\section{References}

[1] Shannon C E 1949 Proc. IRE 3710

See also Whittaker E T 1915 Proc. R. Soc. Edinburgh A 35 181

Goodman J W 1996 Introduction to Fourier Optics 2nd edn (New York: McGraw-Hill)

[2] See, for instance Perelomov A 1986 Generalized Coherent States and their Applications (Berlin: Springer) chapter 1

[3] Schrödinger E 1926 Naturwissenschaften 14664

[4] Klauder J R and Sudarshan E C G 1968 Fundamentals of Quantum Optics (New York: Benjamin)

[5] For detailed expositions see [2] and Klauder J R and Skagerstam B S 1985 Coherent States-Applications in Physics and Mathematical Physics (Singapore: World Scientific). The latter reference includes an excellent survey of the subject and a vast reprint collection.

[6] Shapere A and Wilczek F 1989 Geometric Phases in Physics (Singapore: World Scientific)

[7] von Neumann J 1955 Mathematical Foundations of Quantum Mechanics (Princeton, NJ: Princeton University Press) chapter 5 , section 4
Bargmann V, Butera P, Girardello L and Klauder J R 1971 Rep. Math. Phys. 2221

Perelomov A M 1971 Theor. Math. Phys. 6213

[8] See, for instance Bacry H, Grossmann A and Zak J 1975 Phys. Rev. B 121118

Boon M and Zak J 1976 J. Math. Phys. 192308

Boon M and Zak J 1978 Phys. Rev. B 186744

Janssen A J E M 1982 J. Math. Phys. 23720

[9] Zak J 1967 Phys. Rev. Lett. 191385

Zak J 1968 Phys. Rev. 168686

Ehrenreich, Seitz H and Turnbull D (ed) 1972 Solid State Physics vol 27 (New York: Academic) p 1

[10] Stone M 1930 Proc. Natl Acad. Sci. USA 16172 von Neumann J 1931 Math. Ann. 104570

[11] Mukunda N 1978 Pramana 111

[12] Paley R E A C and Wiener N 1934 Fourier Transforms in the Complex Domain vol 19 (New York: AMS) chapters VI, VII

Levinson N 1940 Gap and Density Theorems vol 26 (New York: AMS) chapter IV

Mukunda N and Sudarshan E C G 1978 Pramana 10227

[13] Boon M and Zak J 1978 Phys. Rev. B 186744 